\title{
Noninvasive Imaging of Human Immune Responses in a Human Xenograft Model of Graft-Versus-Host Disease
}

\author{
Catharina H.M.J. Van Elssen*1, Mohammad Rashidian*2,3, Vladimir Vrbanac ${ }^{* 4}$, Kai W. Wucherpfennig 5,6, \\ Zeina el Habre ${ }^{7}$, Jana Sticht ${ }^{7}$, Christian Freund ${ }^{7}$, Johanne T. Jacobsen ${ }^{2,3,8}$, Juanjo Cragnolini ${ }^{2,3}$, Jessica Ingram ${ }^{2,3}$, \\ Loes Plaisier ${ }^{9}$, Eric Spierings ${ }^{9}$, Andrew M. Tager ${ }^{4,10}$, and Hidde L. Ploegh ${ }^{2,3}$ \\ ${ }^{I}$ Division of Hematology, Department of Internal Medicine, Maastricht University Medical Center, Maastricht, The Netherlands; \\ ${ }^{2}$ Whitehead Institute for Biomedical Research, Cambridge, Massachusetts; ${ }^{3}$ Department of Biology, Massachusetts Institute of \\ Technology, Cambridge, Massachusetts; ${ }^{4}$ Ragon Institute of MGH, MIT and Harvard, Cambridge, Massachusetts; ${ }^{5}$ Department of \\ Cancer Immunology and Virology, Dana-Farber Cancer Institute, Boston, Massachusetts; ${ }^{6}$ Department of Microbiology and \\ Immunobiology, Harvard Medical School, Boston, Massachusetts; ${ }^{7}$ Protein Engineering Group, Leibniz Institute for Molecular \\ Pharmacology and Freie Universität Berlin, Berlin, Germany; ${ }^{8}$ Center for Immune Regulation, Oslo University Hospital, University \\ of Oslo, Oslo, Norway; ${ }^{9}$ Laboratory of Translational Immunology, University Medical Center Utrecht, Utrecht, The Netherlands; and \\ ${ }^{10}$ Center for Immunology and Inflammatory Diseases, Division of Rheumatology, Allergy and Immunology, Massachusetts General \\ Hospital, Charlestown, Massachusetts
}

The immune system plays a crucial role in many diseases. Activation or suppression of immunity is often related to clinical outcome. Methods to explore the dynamics of immune responses are important to elucidate their role in conditions characterized by inflammation, such as infectious disease, cancer, or autoimmunity. Immuno-PET is a noninvasive method by which disease and immune cell infiltration can be explored simultaneously. Using radiolabeled antibodies or fragments derived from them, it is possible to image disease-specific antigens and immune cell subsets. Methods: We developed a method to noninvasively image human immune responses in a relevant humanized mouse model. We generated a camelid-derived single-domain antibody specific for human class II major histocompatibility complex products and used it to noninvasively image human immune cell reconstitution in nonobese diabetic severe combined immune deficiency $\gamma-/-$ mice reconstituted with human fetal thymus, liver, and liver-derived hematopoietic stem cells (BLT mice). Results: We showed imaging of infiltrating immunocytes in BLT mice that spontaneously developed a graft-versus-host-like condition, characterized by alopecia and blepharitis. In diseased animals, we showed an increased PET signal in the liver, attributable to infiltration of activated class II major histocompatibility complex ${ }^{+} \mathrm{T}$ cells. Conclusion: Noninvasive imaging of immune infiltration and activation could thus be of importance for diagnosis and evaluation of treatment of graft-versus-host disease and holds promise for other diseases characterized by inflammation.

Key Words: GvHD; single domain antibodies; ImmunoPET; humanized mice

J Nucl Med 2017; 58:1003-1008

DOI: 10.2967/jnumed.116.186007

Received Oct. 25, 2016; revision accepted Jan. 17, 2017.

For correspondence or reprints contact: Hidde L. Ploegh, Whitehead Institute for Biomedical Research, 9 Cambridge Center, Cambridge, MA 02142.

E-mail: ploegh@wi.mit.edu

${ }^{*}$ Contributed equally to this work.

Published online Feb. 16, 2017.

COPYRIGHT (C 2017 by the Society of Nuclear Medicine and Molecular Imaging.
$\mathbf{P}$ ET makes use of radiolabeled antibodies against disease-specific antigens (1). This approach finds its application most frequently in cancer, where tumors can be visualized by detection of tumorspecific antigens (2). Immuno-PET can assist in diagnosis and disease staging and may have added utility for treatment, because there is often a correlation between antigen expression and clinical effect of anticancer therapy (3). Beyond imaging cancer, there has been an increasing effort to use immuno-PET to assess infectious events (4) or to image infiltration of immune cells (5). The use of fullsized antibodies has its limitations when applied to immuno-PET. Their considerable size $(\sim 150 \mathrm{kDa})$ contributes to a long circulatory half-life and modest tissue penetration. To offset these drawbacks, smaller immunoglobulin-derived formats have been developed, allowing same-day imaging (6).

We and others have developed noninvasive radiolabeled VHHbased immuno-PET imaging to detect immune cell infiltration in mice as a relevant preclinical model (5,7-9). Members of the camelid family (e.g., camels, alpacas), in addition to producing conventional antibodies, make a subset of Igs that are heavy chain-only. These can be reduced in size to small $(\sim 14.5 \mathrm{kDa})$ antibody fragments (termed VHHs) that consist of a single monovalent antibody variable domain, which retains the antigen-binding capabilities of conventional 2-chain antibodies (10). Because of their smaller size, stability, increased tissue penetration, and rapid circulatory clearance, the use of VHHs for imaging has advantages over conventional antibodies (11).

To image human immune cell infiltration and activation in inflammation, we used immunodeficient mice reconstituted with a human immune system. This is achieved through transplantation of NOD/SCID IL2R $\gamma^{\mathrm{c}}$-deficient recipient BLT mice (12). The BLT mouse shows reconstitution of human lymphoid and myeloid lineages. Developing $\mathrm{T}$ cells are educated in the context of the human thymus graft. BLT mice often develop graft-versus-host (GvHD) disease characterized by massive infiltration of activated $\mathrm{T}$ cells (13), albeit with a delay as compared with chronic GvHD in a clinical transplant setting. GvHD is one of the major complications of allogeneic hematopoietic stem cell transplantation, a curative 
immunotherapeutic option for different hematologic neoplasias. GvHD is characterized by $\mathrm{T}$ cell infiltration into target organs, with the most severe effects occurring in the gastrointestinal tract and skin (13). To prevent GvHD, one must maintain a careful balance between $\mathrm{T}$ cell inhibition-accomplished by immunosuppressive drugs - and $\mathrm{T}$ cell activation, to maintain both graft versus tumor effects and prevention of GvHD. We show invasion of activated human $\mathrm{T}$ cells in the mouse liver in the BLT GvHD model. Our results demonstrate the feasibility of detecting active immune responses noninvasively and suggest wider applicability of $\mathrm{VHH}-$ based immuno-PET in a clinical setting.

\section{MATERIALS AND METHODS}

\section{Generation of VHH Specific for Human Class II \\ MHC Molecules}

Procedures for the generation of VHHs have been described in detail previously (14). Briefly, to obtain VHHs specific for human class II MHC, 1 alpaca underwent 5 rounds $(\sim 100 \mu \mathrm{g}$ each) of immunization with purified human leukocyte antigen (HLA)-DRB1*01:01, DRB1*04:01, and DRB $1 * 15: 01$. These proteins were purified using established methods $(15,16)$. After RNA isolation, complementary DNA synthesis of the alpaca heavy chain, and expression in TG1 bacteria for phage display, panning was performed by enzyme-linked immunosorbent assay against purified HLA-DR.

\section{Expression and Purification of HLA-DR1 Proteins}

For nuclear MR (NMR) experiments, recombinant HLA-DR1 proteins were produced from Escherichia coli as previously described $(17,18)$. Briefly, HLA-DR1 $\alpha$ - and $\beta$-chains were expressed separately either unlabeled in $2 \mathrm{YT}$ medium or ${ }^{15} \mathrm{~N}$-labeled in minimal medium (M9) supplemented with ${ }^{15} \mathrm{~N}-\mathrm{NH}_{4} \mathrm{Cl}(1 \mathrm{mg} / \mathrm{mL})$. Inclusion bodies were purified under denaturing conditions by ion exchange chromatography. A labeled and unlabeled chain were combined and refolded together by dilution, to yield ${ }^{15} \mathrm{~N} \alpha$ - or ${ }^{15} \mathrm{~N} \beta$-labeled HLA-DR1, to reduce signal overlap in comparison to a ${ }^{15} \mathrm{~N} \alpha / \beta$-labeled sample. The $\mathrm{HA}_{306-318}$ peptide (PKYVKQNTLKLAT, purchased from Peptides and Elephants) was then loaded onto refolded HLA-DR1 by applying a 10-fold molar excess. Resulting HLA-DR1/HA complexes were further purified by gel filtration in phosphate-buffered saline buffer, pH 5.8 (Superdex 200; GE Healthcare) before NMR measurements.

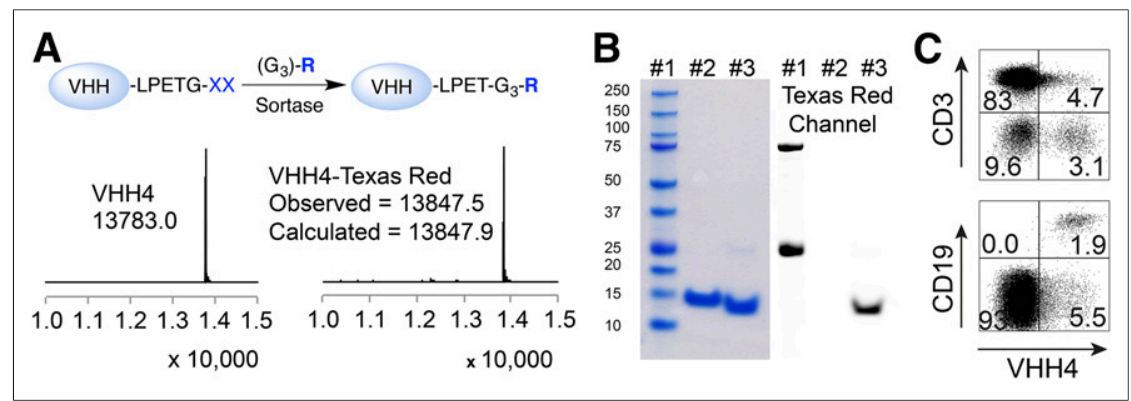

FIGURE 1. Characterization of anti-human class II MHC VHH (VHH4). (A) Schematic representation of sortase reaction to site-specifically labeled VHHs followed by liquid chromatography-mass spectrometry analysis. Sortase recognizes LPXTG motif and replaces $G$ residue with substrate containing a $\mathrm{NH}_{2}-\mathrm{G}_{3}-\mathrm{R}$ moiety, where $\mathrm{R}$ represents tag of interest. Liquid chromatography-mass spectrometry analysis on $\mathrm{VHH} 4$ and $\mathrm{VHH} 4-\mathrm{Texas}$ Red confirms efficient labeling for $\mathrm{VHH} 4$. (B) Sodium dodecyl sulfate polyacrylamide gel electrophoresis characterization of VHH4: lane 1, marker; lane 2, VHH4; lane 3, VHH4-Texas Red (left); and same sodium dodecyl sulfate polyacrylamide gel electrophoresis gel scanned for Texas Red fluorophore (right). (C) Freshly isolated human peripheral blood lymphocytes cells were stained with VHH4-Texas Red and B and T cell lineage-specific antibodies. Representative data of 1 of 3 donors. individual protein complexes $(200 \mu \mathrm{M})$ were performed at $310 \mathrm{~K}$ in sphate-buffered saline buffer ( $\mathrm{pH}$ 5.8), containing $10 \% \mathrm{D}_{2} \mathrm{O}$. Spectra analysis. Chemical shift differences were calculated using the equation $\Delta \delta=\left(\delta \mathrm{H}^{2}+(0.15 \delta \mathrm{N})^{2}\right)^{0.5}$ and were considered as significant if $\Delta \delta$ Goddard and D.G. Kneller, SPARKY 3, University of California) and value minus the $\mathrm{SD}$.

\section{RESULTS}

\section{Development of VHH Specific for Human Class II}

To generate a VHH directed against human class II MHC products, an alpaca was immunized with purified HLA-DRB1*01:01, DRB1*04:01, and DRB1*15:01 (19). Following standard selection and cloning procedures, described in the "Materials and Methods" section, a candidate VHH that showed specific binding to HLA-DR in enzyme-linked immunosorbent assay was identified: VHH4. To further characterize its specificity, VHH4 was conjugated to fluorophore tags in a sortase-mediated reaction (Fig. 1A) (20). Sortase recognizes a LPXTG motif and cleaves the bond between the threonine and glycine, forming a thioester intermediate (21). A Gly3-R the enzyme in the thioester intermediate and forms a protein-LPETGGGR product. Texas Red was conjugated to VHH4 in this manner. iquid chromatography-mass spectrometry and sodium dodecyl and 1B). Although having similar sizes, the fluorophore-labeled (Texas Red) VHH4 migrated faster in sodium dodecyl sulfate polyTexas Red (Fig. 1B).

Alexafluor532-conjugated VHH4 was analyzed for its reactivity with class II HLA antigens by Luminex (Luminex Corp.). The HLA-DRB antigen-coated beads carry the commonly occurring HLA-DRB molecules, establishing that $\mathrm{VHH} 4$ recognizes all human HLA-DR products with the exception of DRB $3 * 01$ (data not shown). The choice of proteins used for alpaca immunization was dictated by availability of purified material in quantities sufficient for screening and immunization. The type of antibodies that emerges from such xenogeneic immunizations often fails to show preferential reactivity with any particular allelic variant and instead broadly cross-reacts with the various allomorphs at that locus.

To investigate the details of HLA-DR1VHH4 interaction, ${ }^{1} \mathrm{H}_{-}{ }^{15} \mathrm{~N}-\mathrm{HSQC}$ spectra of ${ }^{15} \mathrm{~N} \alpha$ - or ${ }^{15} \mathrm{~N} \beta$-labeled HLA-DR1/HA in the absence and in the presence of equimolar amounts of VHH4 were compared (Supplemental Figs. 1 and 2; supplemental materials are available at http://jnm.snmjournals.org). Indicated in Supplemental Figure 1, several 
NMR resonances displayed significant changes in chemical shift, confirming direct interaction of VHH4 with the residues concerned. The amide groups in both chains of the class II MHC molecule were affected. When projected onto the corresponding crystal structure, the affected residues - with the exception of $\alpha \mathrm{Glu} 4, \alpha \mathrm{His} 33$, and $\beta$ Val85-localize to the membrane-proximal $\alpha 2$ and $\beta 2$ domains (Supplemental Fig. 1B), far from the peptide-binding groove. This interface coincides with the interaction site for the CD4 coreceptor, as can be seen from Supplemental Figure 1C, which shows that the VHH4 binding region is also involved in the interaction with the CD4 D1 domain as determined by crystallography. The binding site thus identified is highly conserved and explains the broad reactivity of VHH4 with the many HLA-DR specificities examined. Nonetheless, we cannot exclude the possibility that different allelic HLADRB products might not all bind VHH4 equally well. No chemical shift perturbation was observed when a control VHH (VHH7, recognizing murine Class II MHC products) $(9,22)$ was added in equimolar amounts (Supplemental Fig. 3).

To assess binding of VHH4 to class II MHC molecules at the cell surface, human peripheral blood mononuclear cells were incubated with Alexa647-labeled VHH4 and analyzed by flow cytometry. Resting T cells $(\mathrm{CD} 3+)$ showed no binding to $\mathrm{VHH} 4$, whereas B cells (CD19+) and other class II MHC-positive antigen-presenting cells showed specific staining (Fig. 1C).

\section{Immune Reconstitution of Humanized (Hu)-BLT Mice}

$\mathrm{Hu}$-BLT mice were generated on a NOD-SCID $\gamma-/-$ background by transplantation with human fetal liver and fetal thymus tissue, surgically implanted underneath the kidney capsule. For hematopoietic reconstitution, mice received human fetal liver hematopoietic stem cells. Twenty-week-old mice were analyzed for the presence of human immune cells. Mice received an intravenous injection of

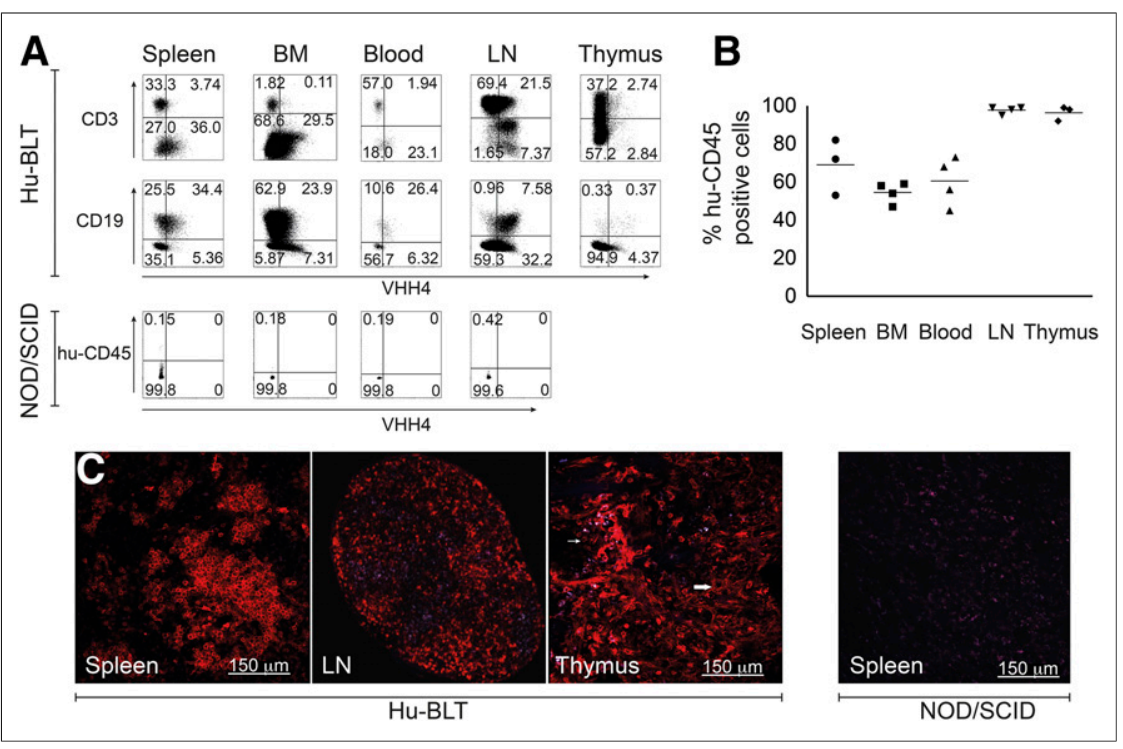

FIGURE 2. Human reconstitution of BLT mice. BLT mice were injected with $10 \mu \mathrm{g}$ of $\mathrm{VHH} 4-$ Texas Red via tail vein injection. Two hours after injection, mice were euthanized and organs isolated. (A) Single cell suspensions were prepared. By flow cytometry, cells were gated on human CD45 and analyzed for expression of class II MHC (VHH4) and B and T cell lineage markers; data representative of 5 individual experiments. (B) Percentages of human CD45-positive cells in different organs as analyzed by flow cytometry. (C) Analysis of VHH4 staining in spleen, lymph node, and thymus by 2-photon microscopy on Hu-BLT mice $(n=3)$ or in spleen of nonreconstituted NOD/SCID mouse, analyzed by 2-photon microscopy $(n=3)$. BM = bone marrow; $L N=$ lymph node.
Texas Red-labeled VHH4 $(10 \mu \mathrm{g})$, followed by excision of the lymphoid organs $2 \mathrm{~h}$ after injection. Flow cytometry results suggested significant reconstitution of a human immune system, with human T cells, B cells, and other class II MHC+ antigen-presenting cells present in mouse spleen, bone marrow, blood, lymph nodes, and thymus (Fig. 2A). As expected, the degree of reconstitution with human cells varied between organs (Fig. 2B). Animals used were preselected based on peripheral blood CD4 T cell number $(>200$ cells $/ \mu \mathrm{L})$. Reconstitution of the individual organs, as assessed by the number of human CD45+ cells, was comparable for the mice used in our flow cytometry experiments (Fig. 2B), rendering the number of peripheral blood human CD4 $\mathrm{T}$ cells a good predictor for the degree of human reconstitution. The specificity of VHH4 for human cells was further confirmed by the absence of staining of the various lymphoid organs of a NOD/SCID mouse that did not receive a transplant, as further confirmed for a wild type BALB/c mouse (data not shown; Fig. 2A).

We next performed 2-photon microscopy of the spleen, thymus, and lymph nodes to assess location of human MHC II+ cells. As for flow cytometry, BLT mice were injected with Texas Red-conjugated VHH4 $(10 \mu \mathrm{g})$. Organs were harvested $2 \mathrm{~h}$ after injection. In the spleen and cervical lymph nodes, germinal center B cells were positive for VHH4 (Fig. 2C). The human thymus showed disperse expression of class II MHC, likely attributable to thymic antigenpresenting cells and class II MHC-positive thymic epithelial cells. Organs of nontransplanted NOD/SCID mice showed no reactivity with VHH4 (Fig. 2C, spleen).

\section{Noninvasive Immuno-PET of Human Immune Reconstitution}

With the exception of peripheral blood, an assessment of the degree of immune reconstitution by cytofluorimetry or histology requires euthanasia of BLT mice. The analysis is limited to the tissues harvested and obviously excludes further experimentation in live mice. To obtain a full-body image in live animals, we examined the presence of human immune cells in different organs by noninvasive immuno-PET. VHH4 was radiolabeled with ${ }^{64} \mathrm{Cu}$, enabled through installation via a sortase reaction of 1,4,7-triazacyclononanetriacetic acid as a metal chelator (Fig. 3A) (9). Mice were given $1.85 \mathrm{MBq}(50 \mu \mathrm{Ci}$; $\sim 5 \mu \mathrm{g}$ ) of radiolabeled $\mathrm{VHH} 4$ and were imaged $2 \mathrm{~h}$ after injection. The time points for imaging were chosen on the basis of our experience with radiolabeled VHHs, where we showed that the circulatory half-life of VHHs is short $(<15 \mathrm{~min})$ and that images acquired $2 \mathrm{~h}$ after injection of the radiolabeled VHHs provide an excellent signal-tonoise ratio (9). We observed a strong PET signal in the spleen (Figs. 3B and 3C) and in the spine, sternum, hip bones, and proximal and distal humerus and femur. These signals represent the presence of human class II MHC-positive cells and were consistently present in all BLT mice imaged. We also observed low nonspecific accumulation of signal in the kidneys and bladder, common VHH-elimination sites (23). PET/CT of untreated NOD/SCID mice (Figs. 3B and 3C) 


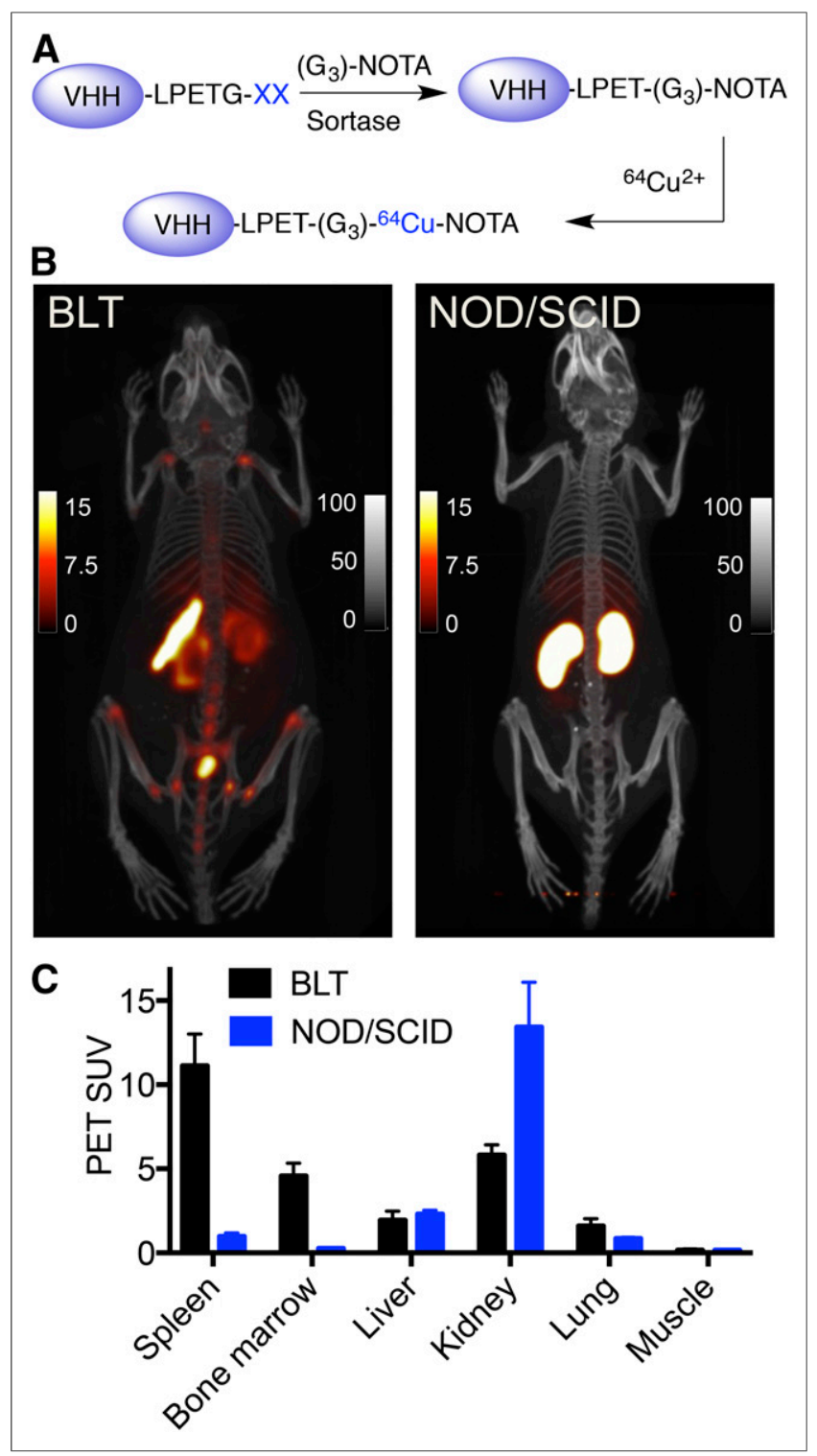

FIGURE 3. PET/CT imaging of human reconstitution in BLT mice using radiolabeled $\mathrm{VHH} 4$. (A) Schematic representation of radiolabeling of $\mathrm{VHHs}$ using sortase. $\mathrm{VHH} 4$ was first labeled using sortase with a chelator molecule, 1,4,7-triazacyclononane-triacetic acid, and then was radiolabeled using ${ }^{64} \mathrm{Cu}$. (B) PET/CT images of BLT mouse (left) and NOD/SCID mouse (right) imaged at $2 \mathrm{~h}$ after injection of ${ }^{64} \mathrm{Cu}-$ $\mathrm{VHH}$ 4. For BLT mouse, PET images show increased signal in spleen and bone marrow. Images are representative data of 4 different BLT mice. For NOD/SCID images, accumulation of signal in kidneys is nonspecific. Images are representative of 2 NOD/SCID mice. (C) PET SUVs of BLT and NOD/SCID mice shown in B. PET scale bars represent SUVs; CT scale bars have arbitrary units. Supplemental Videos 1 and 2 provide better 3-dimensional visualization. Images are all window-leveled to same intensity.

injected with the same amount of ${ }^{64} \mathrm{Cu}$-labeled $\mathrm{VHH} 4$ showed no specific signal (nonspecific uptake in kidneys), further confirming the specificity of $\mathrm{VHH} 4$.

The extent and distribution of lymph nodes varied between different mice, and, if present, only the cervical lymph nodes showed a signal, which is probably due to defects in lymph node development in immunodeficient mice (24). This is in contrast to
BALB/C (data not shown) or B6 mice (9) imaged with a VHH that recognizes mouse class II MHC, which show positive signals for lymph nodes, as well as spleen and thymus.

We concluded that the PET signals obtained with radiolabeled VHH4 in the BLT mice are specific. Radiolabeled VHH4 thus enables detection of human immune reconstitution of class II MHC-positive cells in BLT mice, consistent with the results obtained by flow cytometry and 2-photon microscopy.

\section{Detection of Inflammation in GvHD Model with Liver Involvement}

By a mechanism as yet poorly understood, some BLT mice develop GvHD. This not only is due to the human-mouse MHC mismatch, but also apparently depends on the HLA type of the fetal donor (13). GvHD in these mice may well be provoked by coincident infections and is characterized by human $\mathrm{T}$ cell infiltration in the affected organs (13). Activated human $\mathrm{T}$ cells upregulate expression of class II MHC products (25). We hypothesized that it should be possible to detect such activated, infiltrating $\mathrm{T}$ cells using ${ }^{64} \mathrm{Cu}$-labeled $\mathrm{VHH} 4$ in PET/CT. To explore this possibility, BLT mice with GvHD stage 3 (full body alopecia, Fig. 4A) were imaged by PET/CT using VHH4. We observed an intense PET signal in the liver, not seen in BLT mice without GvHD (stage 0) or control NOD/SCID mice (Figs. 4B and $3 \mathrm{~B}$, respectively).

Two-photon microscopy with fluorophore-labeled VHH4 in GvHD-affected mice showed increased reactivity in the liver (Fig. 4C), which we attribute to inflammation, compared with the liver of a mouse without GvHD (stage 0). To detect the source of the increased class II MHC signal, all organs including liver were dispersed into cell suspensions and analyzed by flow cytometry. We observed increased human $\mathrm{T}$ cell infiltration (Fig. 4D) and an increase in class II MHC-expressing $\mathrm{T}$ cells in samples from liver, consistent with an activated phenotype, not observed in BLT mice without GvHD (Fig. 4E).

\section{DISCUSSION}

The unique characteristics of VHHs make them useful to detect immune reconstitution in BLT mice, not only by conventional invasive methods but also, more importantly, by noninvasive imaging. Here we report the development of a $\mathrm{VHH}$ that recognizes HLA-DR, human class II MHC products. Because class II MHC is expressed on antigen-presenting cells and also marks activated $\mathrm{T}$ cells and natural killer cells, VHH4 was used to detect immune responses (26). In a xenograft model of chronic GvHD, detection of human inflammation, and more specifically the presence of activated human T cells, correlated with clinical GvHD. In this model, it is thus possible to image human immune responses by means of a noninvasive VHH-based PET/CT method.

Reconstitution of a more or less complete human immune system in BLT mice is commonly assessed by flow cytometry and immunohistochemistry $(27,28)$. Human cells are present in blood, bone marrow, spleen, lymph node, liver, intestine, and thymic organoid. However, the percentages of human cells and the extent of reconstitution vary between animals, even within a single reconstitution cohort (28). To improve homogeneity of experimental cohorts, selection of mice with a similar extent and pattern of reconstitution is crucial. Noninvasive imaging can show immune reconstitution of all organs. Accordingly, mice can be selected to more precisely meet the experimental needs. Imaging with VHH4 is obviously limited to providing information on reconstitution of class 


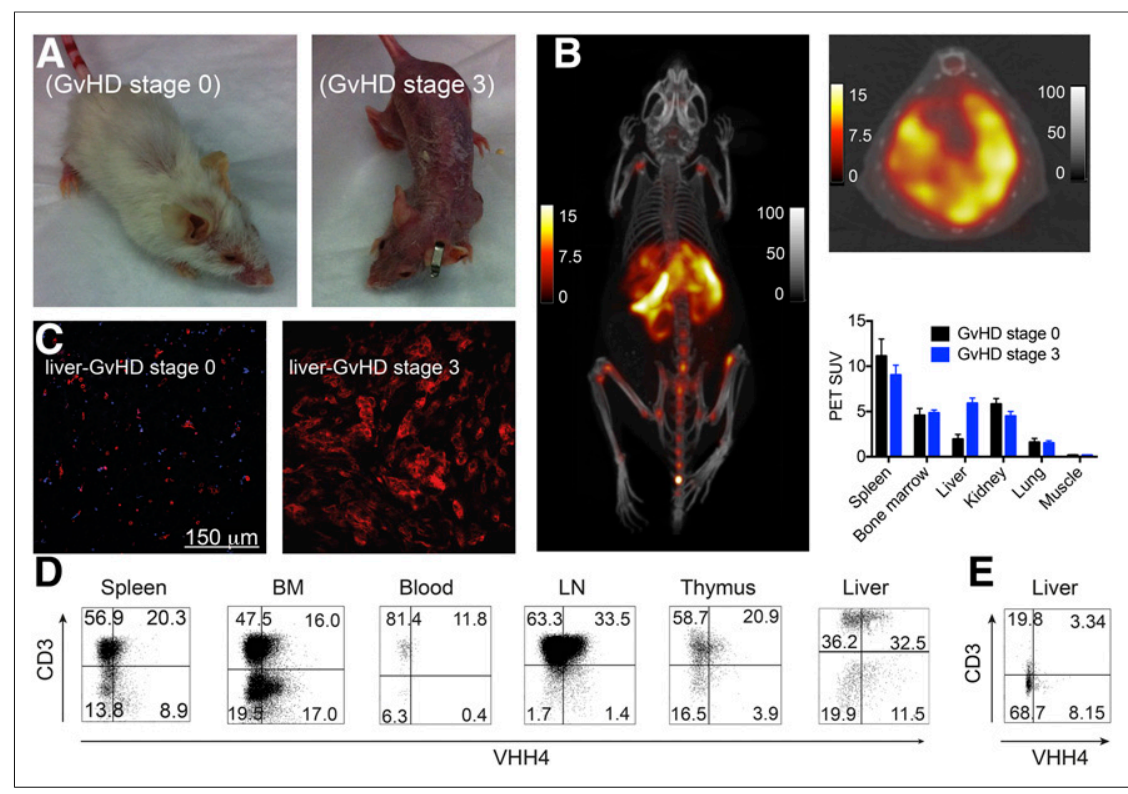

FIGURE 4. Imaging of GvHD in hu-BLT mice using VHH4. Mice with and without clinical symptoms of GvHD were injected with Texas Red-labeled or ${ }^{64} \mathrm{Cu}$-labeled-VHH4. Mice were either euthanized $2 \mathrm{~h}$ after injection to collect organs or imaged by PET/CT. (A) Phenotype of BLT mice with GvHD stage 0 or stage 3. (B) PET/CT images of BLT mouse with stage 3 GvHD $(n=2)$. PET/CT images show intense PET signal in spleen, marrow, and liver. Supplemental Video 3 provides better 3-dimensional visualization. On top right is shown transverse PET/CT image of liver of same mouse for better visualization of signal. PET scale bars represent SUVs; CT scale bars have arbitrary units. Below transverse image is PET SUVs for BLT mice with stage 0 GvHD $(n=2)$ and stage 3 GvHD $(n=2)$ imaged with ${ }^{64} \mathrm{Cu}$-labeled VHH4. (C) Twophoton imaging of the liver of a BLT mouse without clinical sings of GvHD (stage 0) or with stage 3 GvHD. (D and E) Single-cell suspensions of different organs were prepared for mice with GvHD stage $3(\mathrm{D})$ or stage $0(\mathrm{E})$. By flow cytometry, cells were gated on human CD45 and analyzed for expression of human class II MHC and T cell lineage markers. BM = bone marrow; $\mathrm{LN}=$ lymph node.

II MHC-positive immune cells. Other human-specific VHHs would have to be developed to expand the series of markers that can be captured, an eminently feasible proposition.

We were able to noninvasively image infiltration by activated human $\mathrm{T}$ cells in a xenograft model of GvHD. The histopathologic characteristics of chronic GvHD are seen in the BLT model: there is $\mathrm{T}$ cell infiltration in a variety of target organs, inducing inflammation and sclerosis (13). Hu-BLT mice develop a human T cell repertoire that can recognize pathogens and mount immune responses against them (28). It is unclear whether human $\mathrm{T}$ cells undergo positive and negative selection also in the mouse thymus, yielding $\mathrm{T}$ cells restricted by murine MHC products, or whether selection in the human thymus is influenced by mouse antigen-presenting cells that take up residence there. Preliminary indications point in this direction, because the GvHD target organs are infiltrated with human $\mathrm{T}$ cells but lack human antigen-presenting cells (13), making it a suitable xenograft model for GvHD, because it corresponds better to the human situation, within limits.

Immuno-PET of animals with clinical signs of GvHD showed infiltration predominantly of the liver. Previous reports have described $\mathrm{T}$ cell infiltration of the skin, lungs, and bowel as well (13). There are several different possible explanations for our inability to observe $\mathrm{T}$ cell infiltration in the skin, even in animals that show clear skin abnormalities. The signal intensity of hot organs results from high numbers of antigen-positive cells per volume-or density of the antigen molecules-which can bind to radiolabeled VHHs. PET images show massive inflammation in the liver, whereas the lack of signals in other potentially affected organs such as the bowel could be due to a lower density of MHC II+ cells present there, below the limits of PET sensitivity or by scavenging of labeled $\mathrm{VHH}$ by the hot organs. When we injected a far larger dose of Texas Redlabeled VHH4 and examined the skin of GvHD-affected BLT animals by 2-photon microscopy, we observed an increase in the number of VHH4-positive cells compared with skin from mice that did not show clinical GvHD (Supplemental Fig. 4). Because of the sensitivity and resolution of the animal PET used for imaging, detecting PET signals from the thin skin cell layer will remain a challenge. The absence of signal from the intestine and lungs in the BLT mice can also be explained by the fact that these organs are not affected by GvHD in our model, a notion supported by a lack of clinical signs of involvement of these organs (no dyspnea, diarrhea, or weight loss).

\section{CONCLUSION}

Noninvasive detection of human inflammatory responses in the BLT GvHD model suggests the exciting possibility of using VHH-based PET/CT to detect and evaluate the effect of treatment in GvHD. A tumor antigen-specific $\mathrm{VHH}$ (anti-human epidermal growth factor receptor 2) has been used recently in a clinical phase I study for imaging of breast cancer (29), pioneering work that should clear the path for accelerated approval of other VHHs for clinical use. Our results also open the possibility of using VHH-based PET/CT in other conditions characterized by inflammation, such as fevers of undiagnosed origin.

\section{DISCLOSURE}

This work was funded by NIH R01-AI087879-06 (to Hidde L. Ploegh), DP1-GM106409-03 (NIH Pioneer Award to Hidde L. Ploegh), R01-GM100518-04 (to Hidde L. Ploegh), and Lustgarten Foundation (Hidde L. Ploegh). The NMR experiment was supported by a DFG grant (FR 1325/11-1) to Christian Freund. Catharina H.M.J. Van Elssen is a Kootstra Fellow, supported by the MUMC. Mohammad Rashidian is a Cancer Research Institute Irvington Fellow supported by the Cancer Research Institute. No other potential conflict of interest relevant to this article was reported.

\section{ACKNOWLEDGMENTS}

We thank Scott Malstrom and Howard Mak of MIT Koch PET Imaging Facility for technical assistance, Alexander Bradford of MIT Koch peptide facility for assistance with peptide synthesis, and the flow cytometry facility at Whitehead Institute. 


\section{REFERENCES}

1. Wright BD, Lapi SE. Designing the magic bullet? The advancement of immunoPET into clinical use. J Nucl Med. 2013;54:1171-1174.

2. van Dongen GAMS, Visser GWM, Lub-de Hooge MN, de Vries EG, Perk LR. Immuno-PET: a navigator in monoclonal antibody development and applications. Oncologist. 2007;12:1379-1389.

3. Baselga J, Norton L, Albanell J, Kim YM, Mendelsohn J. Recombinant humanized anti-HER2 antibody (Herceptin) enhances the antitumor activity of paclitaxel and doxorubicin against HER2/neu overexpressing human breast cancer xenografts. Cancer Res. 1998;58:2825-2831.

4. Rolle A-M, Hasenberg M, Thornton CR, et al. ImmunoPET/MR imaging allows specific detection of Aspergillus fumigatus lung infection in vivo. Proc Natl Acad Sci USA. 2016;113:E1026-E1033.

5. Tavaré R, McCracken MN, Zettlitz KA, et al. Engineered antibody fragments for immuno-PET imaging of endogenous CD8 + T cells in vivo. Proc Natl Acad Sci USA. 2014;111:1108-1113.

6. Knowles SM, Wu AM. Advances in immuno-positron emission tomography: antibodies for molecular imaging in oncology. J Clin Oncol. 2012;30:3884-3892.

7. Tavaré R, Escuin-Ordinas H, Mok S, et al. An effective immuno-PET imaging method to monitor CD8-dependent responses to immunotherapy. Cancer Res. 2016;76:73-82.

8. Rashidian M, Keliher E, Dougan M, et al. The use of ${ }^{18} \mathrm{~F}$-2-fluorodeoxyglucose (FDG) to label antibody fragments for immuno-PET of pancreatic cancer. ACS Central Science. 2015;1:142-147.

9. Rashidian M, Keliher EJ, Bilate AM, et al. Noninvasive imaging of immune responses. Proc Natl Acad Sci USA. 2015;112:6146-6151.

10. Hamers-Casterman C, Atarhouch T, Muyldermans S, et al. Naturally occurring antibodies devoid of light chains. Nature. 1993;363:446-448.

11. Chakravarty R, Goel S, Cai W. Nanobody: the "magic bullet" for molecular imaging? Theranostics. 2014;4:386-398.

12. Brainard DM, Seung E, Frahm N, et al. Induction of robust cellular and humoral virus-specific adaptive immune responses in human immunodeficiency virusinfected humanized BLT mice. J Virol. 2009;83:7305-7321.

13. Greenblatt MB, Vrbanac V, Vbranac V, et al. Graft versus host disease in the bone marrow, liver and thymus humanized mouse model. PLoS One. 2012;7:e44664.

14. Pardon E, Laeremans T, Triest S, et al. A general protocol for the generation of Nanobodies for structural biology. Nat Protoc. 2014;9:674-693.
15. Anders A-K, Call MJ, Schulze M-SED, et al. HLA-DM captures partially empty HLA-DR molecules for catalyzed removal of peptide. Nat Immunol. 2011;12:54-61.

16. Pos W, Sethi DK, Call MJ, et al. Crystal structure of the HLA-DM-HLA-DR1 complex defines mechanisms for rapid peptide selection. Cell. 2012;151:1557-1568.

17. Schlundt A, Kilian W, Beyermann M, et al. A xenon-129 biosensor for monitoring MHC-peptide interactions. Angew Chem Int Ed Engl. 2009;48:4142-4145.

18. Frayser M, Sato AK, Xu L, Stern LJ. Empty and peptide-loaded class II major histocompatibility complex proteins produced by expression in Escherichia coli and folding in vitro. Protein Expr Purif. 1999;15:105-114.

19. Bochtler W, Maiers M, Bakker JNA, et al. An update to the HLA nomenclature guidelines of the World Marrow Donor Association, 2012. Bone Marrow Transplant. 2013;48:1387-1388.

20. Guimaraes CP, Witte MD, Theile CS, et al. Site-specific C-terminal and internal loop labeling of proteins using sortase-mediated reactions. Nat Protoc. 2013;8:17871799.

21. Chen I, Dorr BM, Liu DR. A general strategy for the evolution of bond-forming enzymes using yeast display. Proc Natl Acad Sci USA. 2011;108:11399-11404.

22. Duarte JN, Cragnolini JJ, Swee LK, et al. Generation of immunity against pathogens via single-domain antibody-antigen constructs. J Immunol. 2016;197:48384847.

23. Chatalic KLS, Veldhoven-Zweistra J, Bolkestein M, et al. A novel ${ }^{111}$ In-labeled anti-prostate-specific membrane antigen Nanobody for targeted SPECT/CT imaging of prostate cancer. J Nucl Med. 2015;56:1094-1099.

24. Legrand N, Weijer K, Spits H. Experimental models to study development and function of the human immune system in vivo. J Immunol. 2006;176:2053-2058.

25. Holling TM, van der Stoep N, Quinten E, van den Elsen PJ. Activated human T cells accomplish MHC class II expression through T cell-specific occupation of class II transactivator promoter III. J Immunol. 2002;168:763-770.

26. Holling TM, Schooten E, Langerak AW, van den Elsen PJ. Regulation of MHC class II expression in human T-cell malignancies. Blood. 2004;103:1438-1444.

27. Hur EM, Patel SN, Shimizu S, et al. Inhibitory effect of HIV-specific neutralizing IgA on mucosal transmission of HIV in humanized mice. Blood. 2012;120:4571-4582.

28. Melkus MW, Estes JD, Padgett-Thomas A, et al. Humanized mice mount specific adaptive and innate immune responses to EBV and TSST-1. Nat Med. 2006;12:13161322.

29. Keyaerts M, Xavier C, Heemskerk J, et al. Phase I study of ${ }^{68}$ Ga-HER2-Nanobody for PET/CT assessment of HER2 expression in breast carcinoma. J Nucl Med. 2016;57:27-33. 